\title{
Seroprevalence and Molecular Epidemiology of HTLV-1 Isolates from HIV-1 Co-Infected Women in Feira de Santana, Bahia, Brazil
}

\author{
Filipe Ferreira de Almeida Rego,,2 Aline Mota-Miranda,,2 Edson de Souza Santos, \\ Bernardo Galvão-Castro,, ${ }^{1,2}$ and Luiz Carlos Alcantara ${ }^{1,2,3}$
}

\begin{abstract}
HTLV-1/HIV-1 co-infection is associated with severe clinical manifestations, marked immunodeficiency, and opportunistic pathogenic infections, as well as risk behavior. Salvador, the capital of the State of Bahia, Brazil, has the highest HTLV-1 prevalence (1.74\%) found in Brazil. Few studies exist which describe this co-infection found in Salvador and its surrounding areas, much less investigate how these viruses circulate or assess the relationship between them. To describe the epidemiological and molecular features of HTLV in HIV coinfected women. To investigate the prevalence of HTLV/HIV co-infection in surrounding areas, as well as the molecular epidemiology of HTLV, a cross sectional study was carried out involving 107 women infected with HIV-1 from the STD/HIV / AIDS Reference Center located in the neighboring City of Feira de Santana. Patient samples were submitted to ELISA, and HTLV infection was confirmed using Western Blot and Polymerase Chain Reaction (PCR). Phylogenetic analysis using Neighbor-Joining (NJ) and Maximum Likelihood (ML) was performed on HTLV LTR sequences in order to gain further insights about molecular epidemiology and the origins of this virus in Bahia. Four out of five reactive samples were confirmed to be infected with HTLV-1, and one with HTLV-2. The seroprevalence of HTLV among HIV-1 co-infected women was $4.7 \%$. Phylogenetic analysis of the LTR region from four HTLV-1 sequences showed that all isolates were clustered into the main Latin American group within the Transcontinental subgroup of the Cosmopolitan subtype. The HTLV-2 sequence was classified as the HTLV-2c subtype. It was also observed that four HTLV/HIV-1 co-infected women exhibited risk behavior with two having parenteral exposure, while another two were sex workers. This article describes the characteristics of co-infected patients. This co-infection is known to be severe and further studies should be conducted to confirm the suggestion that HTLV-1 is spreading from Salvador to surrounding areas.
\end{abstract}

$\mathbf{H}$ UMAN T-CELl Lymphotropic VIRUS TYPE-1 (HTLV-1) is known to be the etiological agent of adult T-cell leukemia/lymphoma (ATL), tropical spatic paraparesis/ HTLV-1 associated myelopathy (TSP/HAM), and other inflammatory diseases. However, the etiology of HTLV-2 remains unclear. While both diseases are endemic worldwide, the rate of seroprevalence differs among regions. ${ }^{1}$

HTLV-1 is endemic in Japan, ${ }^{2}$ regions of Central Africa, ${ }^{3}$ the Caribbean, ${ }^{4}$ and in some areas of Latin America. ${ }^{5}$ Phylogenetic analysis of the most variable DNA region, called a Long Terminal Repeat (LTR) sequence, has classified HTLV-1 into seven subtypes: (a) or cosmopolitan, ${ }^{6}$ (b) or Central African, ${ }^{7}$ (c) or Melanesian, ${ }^{8}$ (d) from Cameroon, ${ }^{9}$ (e) from the Democratic Republic of Congo, ${ }^{10}$ (f) originating from an individual from Gabon, ${ }^{10}$ and (g) a recently described new subtype from Cameroon. ${ }^{11}$ The cosmopolitan subtype can be further divided into five subgroups: (A) Transcontinental; (B) Japanese; (C) West African; (D) North African, and (E) Black Peruvian. 5,12,13,14

HTLV-2 infection is endemic in native populations from North and South America, but is also frequently found in high-risk groups in the United States ${ }^{15}$ and Europe. ${ }^{16-18}$ Phylogenetic analyses have demonstrated that HTLV-2

\footnotetext{
${ }^{1}$ HTLV Center/ Bahia School of Medicine and Public Health/Bahia Foundation for Science Development, Salvador, Bahia, Brazil.

${ }^{2}$ Advanced Public Health Laboratory, Gonçalo Moniz Research Center, Oswaldo Cruz Foundation, Salvador, Bahia, Brazil.

${ }^{3}$ National Cancer Institute, National Institutes of Health, Bethesda, Maryland.
} 
may be classifiable into four subtypes: (a) the predominant variant in the United States and northern Europe, (b) found exclusively in southern Europe, (c) a Brazilian variant of HTLV-2a, and (d) in pygmies from the Democratic Republic of Congo. ${ }^{19}$

HTLV is transmitted via sexual contact, breastfeeding, blood transfusion, and among intravenous drug users, in a similar fashion to the pandemic retrovirus HIV. To understand the consequences of a severe HTLV / HIV co-infection, it is important to evaluate public health policies and treatment options, since both retroviruses are simultaneously present in many regions. In the State of Bahia, the prevalence of HTLV co-infection can reach $20 \%$ in HIV-infected individuals. ${ }^{20}$

Previous studies have suggested that HTLV-1 was brought to the Bahian capital, Salvador, during the slave trade. ${ }^{14,21,22}$ Salvador has the highest HTLV prevalence in the country, $1.35 \%$ among blood donors and $1.8 \%$ in the general population. ${ }^{23,24}$ Previous epidemiological studies have shown that the HTLV-1 strain that is circulating in Bahia appears to be spreading from Salvador to other regions of the state. ${ }^{25,26}$ In an effort to gain further insight into the history of HTLV-1 in Bahia, a molecular epidemiological study was carried out among HIV-1/HTLV co-infected women in the neighboring City of Feira de Santana. This city is located along the route of some of the busiest highways in the State of Bahia $(116 \mathrm{~km}$ from Salvador), at the intersection of three main interstate highways, the BR101, BR116, and BR324. Future studies should be considered in order to ascertain whether new HTLV-1 strains are being brought to Bahia along interstate trucking routes.

This study was approved by the Human Research Ethics Committee of the Bahia School of Medicine and Public Health. Express written consent was obtained from all study participants.

To investigate the seroprevalence and molecular epidemiology of HTLV in Salvador's surrounding regions, a crosssectional study of $107 \mathrm{HIV}-1$ infected women from the STD/ HIV / AIDS Reference Center located in the City of Feira de Santana was carried out.

All blood samples obtained from patients were submitted to ELISA (ORTHO® HTLV-1/ HTLV-1I Ab-Capture ELISA Test Systems, Ortho-Clinical Diagnostics, Raritan, NJ) to select infected individuals, and reactive samples were then submitted to Western blot analysis(HTLV BLOT 2.4; Genelabs Diagnostics ${ }^{\circledR}$, Singapore) for confirmation. After serological testing, patient medical records were analyzed in order to assess age, viral load, CD4 and CD8 T-cell counts, and clinical manifestations in accordance with CDC guidelines, as well as probable transmission route.

Patient DNA was extracted from positive blood samples using a QIAamp® DNA Blood Kit (Qiagen, Alameda, CA).
The DNA was then submitted to HTLV1/2 pol gene nested Polymerase Chain Reaction (PCR) to confirm and distinguish the type of HTLV infection. ${ }^{27}$ A nested-PCR with specific primers for the long terminal repeat (LTR) region of HTLV was performed in order to investigate molecular epidemiology. Two HTLV-1 overlapping fragments were amplified: a LTR-gag segment consisting of 473 base pairs and a tax-LTR segment with 479 base pairs, ${ }^{21}$ and one additional fragment was also amplified to detect the HTLV-2 LTR proviral sequence. $^{28}$ All PCR products were purified using the Qiagen PCR Purification Kit. Sequencing was performed using the Taq FS Dye terminator cycle sequencing kit (Applied Biosystems, Foster City, CA) on an automated 3100 genetic analyzer (Applied Biosystems Inc.) using identical inner primers.

The new LTR sequences, as well as selected reference strains downloaded from the GenBank/EMBL database, were aligned using ClustalX software ${ }^{29}$ and manually edited using GeneDoc software ${ }^{30}$. Phylogenetic analysis was carried out using Neighbor-Joining (NJ) and Maximum-Likelihood (ML) methods in PAUP*, version 4.0b.10. ${ }^{31}$ Tamura-Nei $+\mathrm{g}$ (which takes into account unequal base frequencies, variable transition frequencies, equal transversion frequencies and unequal nucleotide substitution rates among sites) and $\mathrm{HKY}+\mathrm{g}$ (which distinguishes between transitions and transversion rates, unequal base frequencies, and unequal nucleotide substitution rates among sites) evolutionary models were selected for HTLV-1 and HTLV-2 phylogenetic analysis, respectively, using Modeltest 3.7 software. ${ }^{32} \mathrm{NJ}$ tree reliability was assessed by analyzing 1,000 bootstrap replicates. For ML trees, a heuristic search was performed with a subtreepruning-regrafting branch swapping algorithm using the NJ tree as the starting material, including its optimized parameters. Trees were drawn using TreeView software, version $1.4 .^{33}$

The rate of HTLV seroprevalence among HIV-1 infected women was found to be $4.7 \%(n=5)$. Considering the few studies performed with similar objectives, our seroprevalence result is in agreement with the result from another study carried out in the Amazon region that evaluated the coinfection at the same population studied in this report. ${ }^{34}$ Other study carried out in the city of Salvador, have showed a HIV / HTLV seroprevalence of $16.3 \%$ when analyzing the general population. This seroprevalence can not be compared to our result since this rate was estimated in the general population, which increased the prevalence of both infection, separately, and also, this result highlighted the higher prevalence of HIV and HTLV infection in the city of Salvador comparing to Feira de Santana. Out of five reactive samples, four were confirmed to be HTLV-1 and one was confirmed as HTLV-2.

HIV-1 transmission routes were identified as parenteral $(n=2)$ and sexual $(n=3)$. In three of the HTLV-1/HIV-1 co-

Table 1. Age and Probable HIV/HTlV Transmission Route Between HiV-1 Infected and HIV/HTLV Co-infected Women from Feira de Santana, Bahia, Brazil

\begin{tabular}{lcccc}
\hline & & \multicolumn{2}{c}{ Risk exposure } \\
\cline { 3 - 4 } & Age $($ mean $\pm s d)$ & Sexual $(\%)$ & Parenteral $(\%)$ & NI $(\%)$ \\
\hline HIV-1+ HTLV- $(n=102)$ & $38(10,9)$ & $83(81,4 \%)$ & $5(4,9 \%)$ & $14(13,7 \%)$ \\
HIV-1+ HTLV+ $(n=5)$ & $39(6,6)$ & $3(60 \%)$ & $2(40 \%)$ & - \\
\hline
\end{tabular}




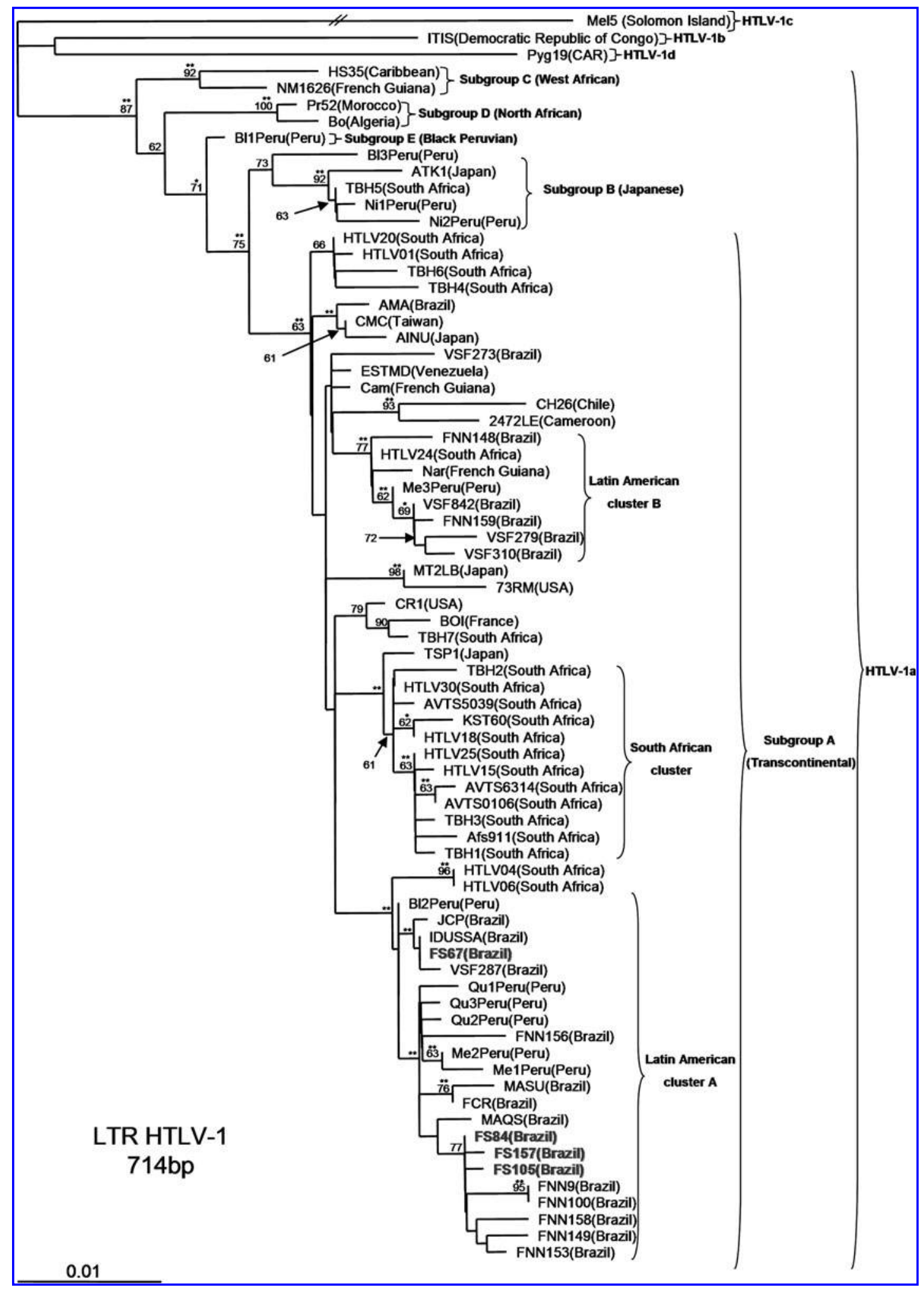

FIG. 1. Rooted neighbor-joining tree of 75 HTLV-1 strains based on a 714-bp fragment of the LTR region. The bootstrap values ( $>60 \%$ using 1000 bootstrap samples) on the branches represent the percentage of trees for which the sequences located at the right end of the branch form a monophyletic group. Mel5 was used as an out-group. Geographic origin is shown in parentheses. Newly sequenced LTRs included in this analysis are shown in bold. Double asterisks $\left(^{* *}\right)$ and single asterisk $\left(^{*}\right)$ signify that the ML method was shown to be highly statistically significant $(p<0.001)$ or statistically significant $(p<0.005)$, respectively. 


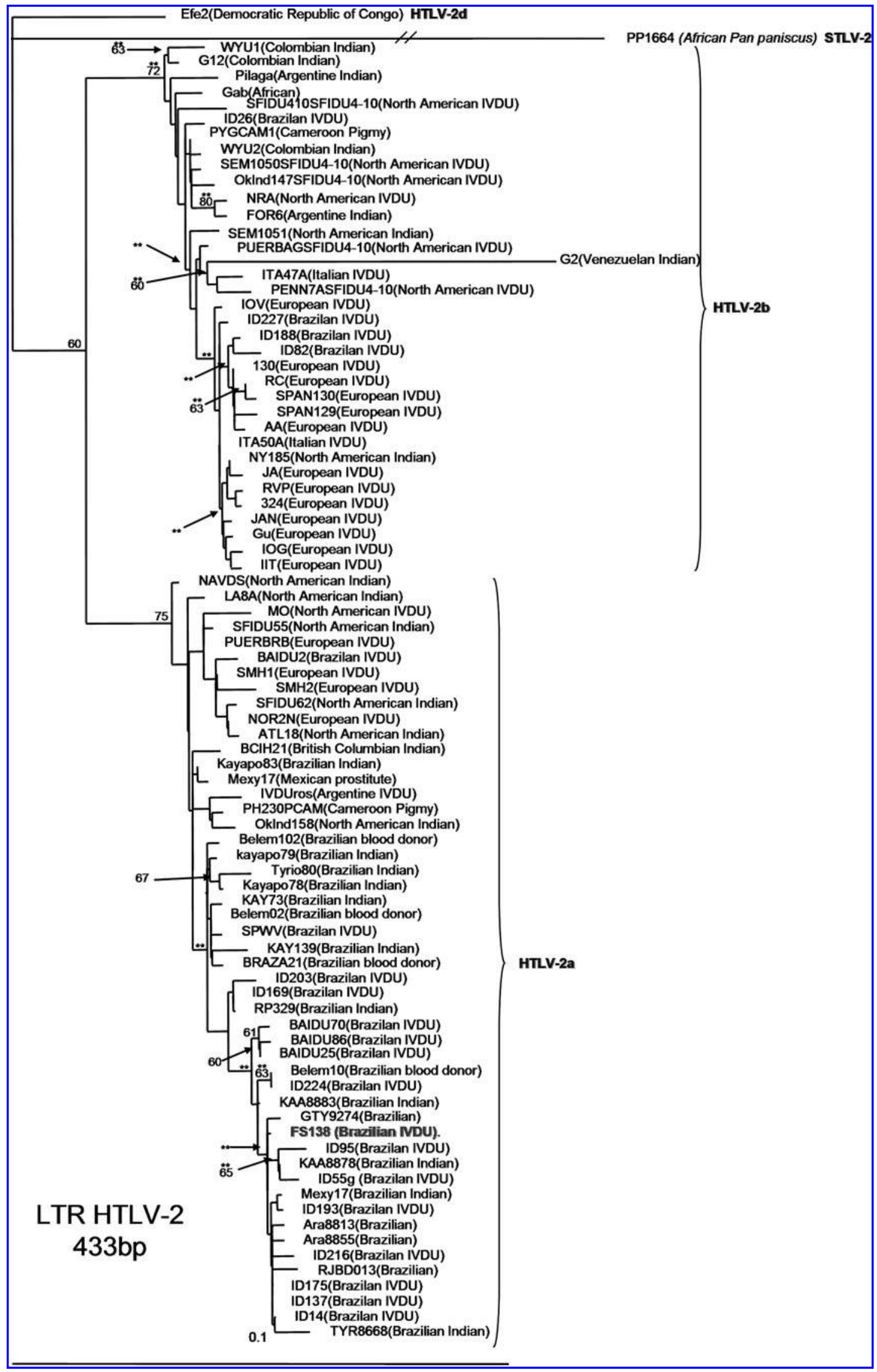

FIG. 2. Rooted neighbor-joining tree of 87 HTLV-2 strains based on a 433-bp fragment of the LTR region. The bootstrap values ( $>60 \%$ using 1000 bootstrap samples) on the branches represent the percentage of trees for which the sequences located at the right end of the branch form a monophyletic group. PP1664 was used as out-group. Geographic origin and risk factor are shown in parentheses. Newly sequenced LTRs included in this analysis are shown in bold. Double asterisks $\left.{ }^{* *}\right)$ signify that the ML method was shown to be highly statistically significant $(p<0.001)$. 
infected women, risk behavior was noted: one received a blood transfusion, while two were sex workers. The woman infected with HTLV-2 was an IVDU for a period of 5 years while living in São Paulo. Co-infection was shown to be a condition that is associated with high-risk groups (Table 1). STD prevention campaigns play an important role in disease prevention since the most prevalent transmission route among HIV-1 co-infected women in Feira de Santana has been observed to be sexual transmission. With regard to CD4 and CD8 T-cell counts, viral load, and clinical manifestations according to CDC guidelines, it was observed that the HTLV/ HIV co-infected group closely resembled the HIV infected patients who were not reactive to HTLV serological screening. All HIV-1 infected individuals included in this study were on antiretroviral treatment, however it is not possible yet to estimate the possible impact of the HAART therapy in the outcome of HIV/HTLV co-infection, since the few studies conducted with this objective are still controversial. ${ }^{35,36}$

Phylogenetic analysis of sequences from the entire LTR region classified all HTLV-1 isolates as belonging to the Transcontinental subgroup of the Cosmopolitan subtype, supported by a bootstrap value of $63 \%(p<0.001$ for ML analyses) (Fig. 1). All of these sequences were grouped in the Latin American cluster A with ML statistical support $(p<0.001)$. Three of the sequences were clustered together with other sequences from Salvador downloaded from the GenBank/EMBL database with bootstrap support of $77 \%$. The FS67 isolate was grouped with other Brazilian sequences, and a $10 \mathrm{bp}$ deletion was observed between the first and second 21 base-pair repeat at 3U-LTR at position 130 and 139 of ATK genome (Accession number: J02029).

Phylogenetic analysis of the HTLV-2 LTR region confirmed that the HTLV-2 isolate belongs to subtype IIa (Fig. 2), supported by a bootstrap value of $75 \%$. This isolate formed a unique cluster inside subtype IIa along with other Brazilian sequences (Brazilian variant HTLV-2c), which was well supported by the ML algorithm. According to another study, ${ }^{37}$ these data suggest the unique introduction of HTLV-2 into this population, as previously associated with high-risk groups, ${ }^{20}$ since HTLV-2 is predominantly transmitted parenterally among IVDUs. However, it is necessary to analyze more patients in order to obtain additional information regarding the transmission of HTLV-2.

This study is in agreement with findings from previous studies, ${ }^{21,22,25,26,38}$ reaching the conclusion that the HTLV-1a Transcontinental subgroup is the most prevalent strain found in Brazil. The presence of HTLV-1 LTR sequences from Bahia in several clusters along the phylogenetic tree suggests multiple post-Columbian introductions of HTLV-1 into the Brazilian population. These data, in addition to other studies from regions throughout Bahia, ${ }^{25,26}$ serve to confirm the postColumbian introduction hypothesis of HTLV-1 into Bahia.

HTLV-1 transcription is regulated by elements in the LTR region, characterized as proviral promoter. ${ }^{39}$ HTLV-1 transcription is activated by the interaction of the viral Tax protein with other transcription factors, forming a CREB-Tax-CBP complex that binds to the third 21-base pair repeated sequence located within the U3-LTR region named TxRE. ${ }^{40}$ Moreover, previous studies have revealed that just two direct repeats of the 21-bp sequence are sufficient for efficient activation by $\mathrm{Tax}^{39}$, and that deletions in the TxRE2S sequence significantly decrease HTLV-1 Tax-mediated transcriptional activity. ${ }^{41}$ This study identified a new $10 \mathrm{bp}$ deletion between the first and second 21 base-pair repeat at 3U-LTR. However, in order to make any inferences about this deletion effect, further functional studies need to be performed.

Recent publications ${ }^{21,22,25,26}$ have suggested that the introduction of the Transcontinental subgroup is probably the result of the Bantu population's migration over the last 3000 years from Central Africa to Southern Africa, giving rise to the Zulu population, or perhaps due to an even more recent migration (fewer than 300 years ago) to the gold mines in South Africa, then eventually to the State of Bahia. This theory appears to be consistent with what has been observed in one sequence from a region in Central Africa and another from Chile, which shares a common ancestor that is segregated within the Latin American cluster B, also containing a sequence from South Africa in its tree topology. Africans from Bantu-speaking areas were the first slaves that were transported in great numbers to Bahia, bringing with them a strong cultural heritage largely preserved in Bahian folklore, religion, language, cuisine, and customs. Although most enslaved Africans were brought from West Africa, there is evidence that many were also brought from other regions of southern Africa. Therefore, South Africans who had been infected could have introduced the HTLV-1 Cosmopolitan subtype of the Transcontinental subgroup into Salvador and possibly into other areas on the Latin American continent.

\section{Sequence Data}

The GenBank accession numbers of the new HTLV-1 fragments included in phylogenetic study were as follows: FJ853491, FS84; FJ853490, FS105; GU225731, FS67; GU225733, FS138; GU225732, and FS157.

\section{Acknowledgments}

Bioinformatics analysis was performed at the LASP/ CPqGM/FIOCRUZ Bioinformatics Unit, supported by FAPESB (Grants 303/03) and the Brazilian Ministry of Health (306/04 and 307/04). The authors are grateful to Noilson Lázaro de Souza Gonçalves and Elisabeth Deliege for their technical assistance.

\section{Author Disclosure Statement}

No competing financial interests exist.

\section{References}

1. Catalan-Soares B, Proietti FA, and Carneiro-Proietti AB. Heterogeneous distribution of HTLV-1/II prevalence rates in blood donors from urban areas in Brazil. Vox Sanguinis 2004;87:105.

2. Kajiyama W, Kashiwagi S, Nomura H, Ikematsu H, Hayashi $\mathrm{J}$, and Ikematsu W. Seroepidemiologic study of antibody to adult T-cell leukemia virus in Okinawa, Japan. Am J Epidemiol 1986;123:41-47.

3. Verdier M, Bonis J, and Denis FA. The prevalence and incidence of HTLV in Africa. AIDS in Africa 1994;9:173-193.

4. Blattner WA, Saxiger DC, Riedel B, et al.: A study of HTLV-I and its associated risk factors in Trinidad and Tobago. J Acquir Immune Defic Syndr Hum Retrovirol 1990;3:11021108. 
5. Proietti FA, Carneiro-Proietti ABF, Catalan-Soares BC, and Murphy EL. Global epidemiology of HTLV-I infection and associated diseases. Oncogene 2005;24:6058-6068.

6. Miura T, Fukunaga T, and Igarashi T. Phylogenetic subtypes of human T-lymphotropic virus type I and their relations to the anthropological background. Proc Natl Acad Sci USA 1994;91:1124-1127.

7. Vandamme A-M, Liu H-F, P. Goubau P, and Desmyter J. Primate T-lymphotropic virus type I LTR sequence variation and its phylogenetic analysis: Compatibility with an African origin of PTLV-I. Virology 1994;202:212-223.

8. Gessain A, Yanagihara R, Francini G, et al.: Highly divergent molecular variants of human T-lymphotropic virus type from isolated populations in Papua New Guinea and the Solomon Islands. Proc Natl Acad Sci USA 1991;88: 7694-7698.

9. Chen J, Zekeng L, Yamashita M, et al.: HTLV isolated from a Pygmy in Cameroon is related but distinct from the known Central African type. AIDS Res Hum Retroviruses 1995; 11:1529-1531.

10. Salemi M, Van Dooren S, Audenaert E, et al.: Two new human T-lymphotropic virus type I phylogenetic subtypes in seroindeterminates, a Mbuti pygmy and a Gabonese, have closest relatives among African STLV-I strains. Virology 1998;246:277-287.

11. Wolfe ND, Heneine W, Carr JK, et al.: Emergence of unique primate T-lymphotropic viruses among central African bushmeat hunters. Proc Natl Acad Sci USA 2005;102:7994-9.

12. Gasmi M, Farouqi B, d'Incan M, and Desgranges C. Long terminal repeat sequence analysis of HTLV type I molecular variants identified in four North African patients. AIDS Res Human Retroviruses 1994;10:1313-1315.

13. Vidal AU, Gessain A, Yoshida M, Tekaia F, Garin B, Guillermain B, Schuls T, Farid R, and De The G. Phylogenetic classification of human $\mathrm{T}$ cell leukaemia/lymphoma virus type I genotypes in five major molecular and geographical subtypes. J Gen Virol 1994;75:3655-3666.

14. Van Dooren, S, Gotuzzo E, Salemi M, et al.: Evidence for a Post-Colombian introduction of human T-cell lymphotropic virus in Latin America. J Gen Virol 1998;79:2695-2708.

15. Khabbaz RF, Onorato IM, Cannon RO, et al.: Seroprevalence of HTLV-1 and HTLV-2 among intravenous drug users and persons in clinics for sexually transmitted diseases. $\mathrm{N} \mathrm{Engl} \mathrm{J}$ Med 1992;326:375-380.

16. Tedder RS, Shanson DC, Jeffries DJ, et al.: Low prevalence in the UK of HTLV-I and HTLV-II infection in subjects with AIDS, with extended lymphadenopathy, and at risk of AIDS. Lancet 1984;2:125-128.

17. Zella D, Mori L, Sala M, et al. HTLV-II infection in Italian drug abusers. Lancet 1990 Sep 1;336(8714):575-6.

18. Vignoli C, Zandotti C, De Lamballerie X, et al.: Prevalence of HTLV-II in HIV-1-infected drug addicts in Marseille. Eur J Epidemiol 1993;9:351-352.

19. Vandamme AM, Salemi M, Van Brussel M, et al.: African origin of human T-lymphotropic virus type 2 (HTLV-2) supported by a potential new HTLV-2d subtype in Congolese Bambuti Efe Pygmies. J Virol 1998;72:4327-4340.

20. Brites C, Harrington Jr WJ, Pedroso C, Netto EM, and Badaró R. Epidemiological characteristics of HTLV-I and II coinfection in Brazilian subjects infected by HIV-1. Braz J Infect Dis $1997 ; 1: 43-48$.

21. Alcantara LCJ, Oliveira T, Gordon M, et al.: Tracing the origin of Brazilian HTLV-1 as determined by analysis of host and viral genes. AIDS 2006;20:780-782.
22. Mota ACA, Van-Dooren S, Fernandes FM, et al.: The close relationship between South African and Latin American HTLV-1 strains corroborated in a molecular epidemiological study of the HTLV-1 isolates from a blood donor cohort. AIDS Res Hum Retroviruses 2007;23:503-507.

23. Galvão-Castro B, Loures L, Rodrigues LGM, et al.: Geographic distribution of human T lymphotropic virus type-I among blood donors: A Brazilian nationwide study. Transfusion 1997;37:242.

24. Dourado I, Alcantara LCJ, Barreto ML, Teixeira MG, and Galvão-Castro B. HTLV-I in the general population of Salvador, Brazil: A city with African ethnic and sociodemographic characteristics. J Acquir Immune Defic Syndr 2003;34:527-531.

25. Rego FF, Alcantara LC, Moura Neto JP, et al.: HTLV type 1 molecular study in Brazilian villages with African characteristics giving support to the post-Columbian introduction hypothesis. AIDS Res Hum Retroviruses 2008;24: 673-677.

26. Magalhães T, Mota-Miranda AC, Alcantara LC, et al.: Phylogenetic and molecular analysis of HTLV-1 isolates from a medium sized town in northern of Brazil: Tracing a common origin of the virus from the most endemic city in the country. I Med Virol 2008;80:2040-2045.

27. Vallejo A and García-Sáiz A. Typing human T-cell lymphotropic virus (HTLV-I and HTLV-II) by nested polymerase chain reaction: Application to clinical specimens. I Virol Methods 1995;51:9-17.

28. Eiraku N, Novoa P, da Costa Ferreira M, et al.: Identification and characterization of a new and distinct molecular subtype of human T-cell lymphotropic virus type 2. J Virol 1996; 70:1481-1492.

29. Thompson JD, Gibson TJ, Plewniak F, et al.: The CLUSTAL_X Windows interface: Flexible strategies for multiple sequence alignment aided by quality analysis tools. Nucleic Acids Res 1997;25:4876-4882.

30. Nicholas KB, Nicholas HBJ, and Deerfield DW. GeneDoc: Analysis and visualization of genetic variation. Embnew News 1997;4:14.

31. Swofford DL, Olsen GJ, Waddell PJ, and Hillis D.M. Phylogenetic inference. Mol System 1996;2:407-514.

32. Posada D and Crandall KA. MODELTEST: Testing the model of DNA substitution. Bioinformatics 1998;14:817-818.

33. Page RDM. TreeView: An application to display phylogenetic trees on personal computers. Cabios Applications Note 1996;12:357-358.

34. Laurentino RV, Lopes IG, Azevedo VN, et al:: Molecular characterization of human T-cell lymphotropic virus coinfecting human immunodeficiency virus 1 infected patients in the Amazon region of Brazil. Mem Inst Oswaldo Cruz 2005; 100:371-376.

35. Machuca A, Rodés B, and Soriano V. The effect of antiretroviral therapy on HTLV infection. Virus Res 2001;78: 93-100.

36. Beilke MA, Traina-Dorge VL, Sirois M, et al.: Relationship between human $\mathrm{T}$ lymphotropic virus (HTLV) type $1 / 2$ viral burden and clinical and treatment parameters among patients with HIV type 1 and HTLV-1/2 co-infection. Clin Infect Dis 2007;44:1229-1234.

37. Alcantara LC, Shindo N, Van Dooren S, et al.: Brazilian HTLV type 2a strains from intravenous drug users (IDUs) appear to have originated from two sources: Brazilian Amerindians and European/North American IDUs. AIDS Res Hum Retroviruses 2003;19:519-523. 
38. Kashima S, Alcantara LCJ, Takayanagui OM, et al:: Distribution of human T cell lymphotropic virus type 1 (HTLV1) subtypes in Brazil: Genetic characterization of LTR and tax region. AIDS Res Hum Retroviruses 2006;22:953-959.

39. Dan S, Tanimura A, and Yoshida M. Interaction of Gli2 with CREB protein on DNA elements in the long terminal repeat of human T-cell leukemia virus type 1 is responsible for transcriptional activation by tax protein. J Virol 1999;73: 3258-3263.

40. Lemasson I, Polakowski NJ, Laybourn PJ, and Nyborg JK. Transcription regulatory complexes bind the human T-cell leukemia virus $5^{\prime}$ and $3^{\prime}$ long terminal repeats to control gene expression. Mol Cell Biol 2004;24:6117-6126.
41. Tanimura A, Dan S, and Yoshida M. Cloning of novel isoforms of the human Gli2 oncogene and their activities to enhance tax-dependent transcription of the human T-cell leukemia virus type 1 genome. J Virol 1998;72:3958-3964.

Address correspondence to: Luiz Carlos Junior Alcantara, PhD NIH-NCI, Vaccine Branch Building 41, Room C303 41 Library Drive, MSC 5055 Bethesda, MD 20892

E-mail: alcantaralc@mail.nih.gov 

This article has been cited by:

1. Galetto Leonardo Ramos, Lunge Vagner Ricardo, Béria Jorge Umberto, Tietzmann Daniela Cardoso, Stein Airton Tetelbom, Simon Daniel. 2014. Short Communication: Prevalence and Risk Factors for Human T Cell Lymphotropic Virus Infection in Southern Brazilian HIV-Positive Patients. AIDS Research and Human Retroviruses 30:9, 907-911. [Abstract] [Full Text HTML] [Full Text PDF] [Full Text PDF with Links]

2. Mahmoud Reza Azarpazhooh, Kazem Hasanpour, Mohsen Ghanbari, S.A. Rahim Rezaee, Baratali Mashkani, Mohammad Reza Hedayati-Moghaddam, Narges Valizadeh, Reza Farid Hosseini, Mohsen Foroghipoor, Azadeh Soltanifar, Maryam Sahebari, Keyhan Azadmanesh, Gholahossein Hassanshahi, Houshang Rafatpanah. 2012. Human T-Lymphotropic Virus Type 1 Prevalence in Northeastern Iran, Sabzevar: An Epidemiologic-Based Study and Phylogenetic Analysis. AIDS Research and Human Retroviruses 28:9, 1095-1101. [Abstract] [Full Text HTML] [Full Text PDF] [Full Text PDF with Links] 